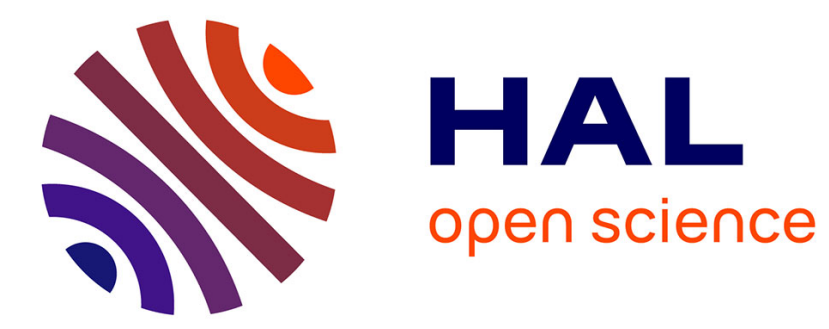

\title{
Gödel, Carnap and the Fregean Heritage
}

Gabriella Crocco

\section{To cite this version:}

Gabriella Crocco. Gödel, Carnap and the Fregean Heritage. Synthese, 2003, 137 (1-2), pp.21-41. halshs-00095781

\section{HAL Id: halshs-00095781 https://shs.hal.science/halshs-00095781}

Submitted on 21 Feb 2017

HAL is a multi-disciplinary open access archive for the deposit and dissemination of scientific research documents, whether they are published or not. The documents may come from teaching and research institutions in France or abroad, or from public or private research centers.
L'archive ouverte pluridisciplinaire HAL, est destinée au dépôt et à la diffusion de documents scientifiques de niveau recherche, publiés ou non, émanant des établissements d'enseignement et de recherche français ou étrangers, des laboratoires publics ou privés. 
Gödel, Carnap and the Fregean Heritage

\author{
Gabriella Crocco \\ Aix Marseille Université \\ CEPERC Aix-en-Provence \\ email: gabriella.crocco@univ-amu.fr
}

Publié dans G. Crocco Synthese, 137, n¹-2, 2003, p 21-41. 


\section{Introduction}

In this paper we examine Gödel's and Carnap's conception of logic and the content of mathematics. This examination will be mainly conducted through the analysis of $I s$ mathematics syntax of language?, the paper that was prepared by Gödel in six versions for the Schilpp's volume dedicated to Carnap, and that in the end was not sent to the editors ${ }^{1}$.

Such an examination is essential in order to evaluate the importance of logic and language in knowledge. Carnap and Gödel are amongst the most influential logicians which have developed the fregean heritage in different and even opposing directions. To compare their doctrines about the role of logic and its status is one of the fundamental steps for an evaluation of the philosophy of the last century .

Such an examination is necessary considering the radical general misunderstanding of Gödel's argument against Carnap. The commentaries given by the two mains editors of the different versions of Gödel's paper (W. Goldfarb and R. Consuegra) simply demonstrate a deep misunderstanding of Gödel's position.

The first main criticism affirms that Gödel is more positivistic than Carnap in Logical Syntax of Language (LSL). For Carnap since 1934 there is no notion of empirical fact prior to any linguistic framework. On the opposite Gödel continues to affirm a very oldfashioned conception of empirical facts, as fixed independently of theoretical frameworks. Moreover he presupposes the "given" as a pure fact even in the field of mathematics, so showing his subordination to a concept of science typical of the logical positivism ${ }^{2}$. We will suggest on this matter a rather different analysis, taking as a starting point the question of the nature of mathematics and logic after paradoxes: Carnap is, as is Frege, a universalist ${ }^{3}$, believing that language is the universal medium between us and "the world". Gödel does not follow Frege on this ground.

The second main criticism of Is mathematics syntax of language? affirms that Gödel had not understood the novelty of Carnap's LSL. Gödel had continued to consider Carnap's work in a foundationalist and reductionist perspective. Such foundationalism was abandoned by Carnap even before $1934^{4}$. We will show, through a careful analysis of version III of Gödel's paper, that there is no justification for the accusation that Gödel

\footnotetext{
${ }^{1}$ Versions III and V of this paper are in the third volume of Gödel's Collected Works [Gödel CWIII] ; versions II and IV were published by R. Consuegra [Consuegra]

${ }^{2}$ See Goldfarb [Gödel CWIII], page 328 and page 333. [Consuegra] page 98.

${ }^{3}$ We take this term in the sense precised by J. Van Heijenoort.

${ }^{4}$ Goldfarb [Gödel CWIII], pages 329, 331, 332. [Consuegra], especially pages 68-69.
} 
overlooked the point. Gödel does not confine himself to repeating the finitist dogma on the nature of syntax, or the foundationalist dogma on the necessity of giving a foundation or a justification of mathematics.

The paper will be organised in two sections.

Firstly, we will briefly sketch Gödel and Carnap's main theses about logic and mathematics, making their respective relationships to Frege's conception of logic explicit. The Fregean heritage has been developed in two alternative and opposing directions: Gödel's solution reintroduces intuition to grasp logical and mathematical objects. Carnap's solution maintains language as the only condition of possibility for mathematical and logical knowledge, but he refuses to acknowledge any logical objects or concepts with any non-conventional content. Both are far from subscribing to a naive, simplistic and reductionist conception of science as it has been suggested by a part of the "folk history of analytic philosophy" 5 and by the vulgate of quinean "Two dogmas of empiricism".

Secondly we analyse Gödel's paper again, in particular version III, in order to restate Gödel's argument. It will appear, from Gödel's analysis, that Carnap's conventionalist solution is unacceptable even from a non-foundationalist point of view. Carnap succeeded in giving an explication of mathematics as language, but uder such an explication mathematical remains a mystery.

\section{Gödel and Carnap's main theses about logic and mathematics}

\subsection{Paradoxes and Logic : Frege}

Let's begin with paradoxes. Frege's naive conception of concepts and of sets can be represented by three theses:

1) Each predicate (the name of a concept) denotes a concept to which corresponds its extension (its range of values). This latter

a) is the set of all objects satisfying the concept

b) is obtained as a partition in the totality of all beings.

2) Every concept is entirely determined by the set of objects constituting its extension.

3) Concepts and sets have an objective existence.

Some points have to been stressed about these assertions.

\footnotetext{
${ }^{5}$ Alberto Coffa has the merit to have destroyed the first, by an attentive analysis of Carnap's works, the "misleading, simplistic, picture which has become part of the folk-history of analytic philosophy" [Coffa] page 547.Carnap, Tarski and the search of truth, Noûs 21, 1987.
} 
Concerning 3), we have to understand the term "existence" in a logical and in an ontological sense. Concepts and objects (and then ranges of values among them) are logical primitives that cannot be defined in an explicit way ${ }^{6}$. Nevertheless, they can be isolated through an analysis of propositions. That is : we can unambiguously apprehend the logical primitives, through an analysis of what language allows us to express. Concepts and objects have a logical and an ontological existence. In the first sense they exist as we can use them as subjects of predication; in the second sense they exist because they conform to laws ${ }^{7}$. If existence reduces to conformity to laws are we allowed to say that concepts and objects are independent of our constructions and intuitions? The answer is yes if we use the term law in the Fregean sense. A law is a true proposition; what is true is such independently of our capacity to recognise it; true propositions are, were and will ever be true; true propositions of mathematics are then necessary, unconditional and eternal ${ }^{8}$.

Concerning 1). The straight correspondence between concepts and sets is the key to the logicistic program. It shows the productivity of logic and the inexhaustibility of mathematics. Logic is not barren, as it allows us to apprehend logical objects, that is range of values; multiplicity taken as one object. The apprehension of concept and logical objects through language frees concepts from sense perception and Kantian pure intuition. Mathematics is inexhaustible because concepts can be of second, third and whatever order we want. Concepts are not condemned to apply only to individual objects ${ }^{9}$.

Concerning 2). The thesis does not only state that a set (or in Fregean term a range of values) is determined exclusively by its elements. The denotation of a concept, for Frege, is not the corresponding class, but, in virtue of Frege's ontological opposition between concepts and objects, the concept itself. Concepts are these strange entities described in "Funktion und Begriff". We cannot speak of concepts without transforming them into something else, that is objects. But, if so, how could we state the identity of two concepts? One way should be to consider senses, which allow us to identify concepts as denotations; Frege simply rejects such a solution in favour of an extentionalist one. This is clearly affirmed in "Comments on Sense and Denotation" :

A concept-word means a concept, if the word is used as it is appropriate for logic. I may clarify this by drawing attention to a fact that seems to weigh heavily on the side of extensionalist as against intensionalist logicians: namely, that in any sentence we can substitute salva veritate one concept-

\footnotetext{
${ }^{6}$ For ex. Frege's "Funktion und Begriff", §24. [Frege KS]

${ }^{7}$ See for ex. Frege Grundlagen der Aritmetik, section 61 [Frege GL]

${ }^{8}$ See for ex. Frege Posthumous Writings, the first and the second version of the "Logik" [Frege PW], or the introduction to the Grundgesetze especially pages XV-XVIII, [Frege GG]

${ }^{9}$ Grundlagen Section $§ 88$.
} 
word for another if they have the same extension, so that [...] concepts differ only in so far their extensions are different." 10

Intensions (senses) cannot be reduced to extensions, but logic is primarily occupied by extensions or more generally by denotations.

"[Extensionalist logicians] are right when they show by their preference for the extension, as against the intension, of a concept that they regard the denotation and not the sense of words as the essential thing for logic. The intensionalist logicians [...] forget that logic is not concerned with how thoughts, regardless of truth-value, follow from thoughts, that the step from thought to truth-value-more generally, the step from sense to denotation- has to be taken. They forget that the laws of logic are first foremost laws in the realm of denotation and only relate indirectly to sense.". ${ }^{11}$

It's a well known fact that 1-3 together are paradoxical; paradoxes concerning the notion of concept (the intensional paradoxes as communicated by Russell to Frege in his letter of 29 September 1902) and the notion of set (the most known paradoxes of sets). Then consequently, in the framework of Frege analysis :

a) there are predicates to which no concept corresponds

b) there are concepts to which no set corresponds

The departure of Carnap and Gödel from Frege can be oversimplified through their respective departure from thesis 1-3.

\subsection{Logic and mathematics: Carnap}

Carnap does accept thesis 1) with some important modifications: concepts are senses whose denotations are sets which are defined through the iterative notion of set. Eliminating concepts from denotations, Frege's extensionalist point of view (thesis 2) will even be reinforced by a formal reduction of intensions (or senses) to extensions, via the notion of logical equivalence ${ }^{12}$.

Moreover Carnap totally rejects 3). He uses sets (classes) as possible arguments for predicates, but all through his philosophical evolution he denies them any kind of independent existence. Carnap was constant in holding such a thesis, but it is really from $L S L$ onward that from a nominalistic attitude he shifts toward a conventionalistic one. In the reductionist and constitutional perspective of the Aufbau (1928) Carnap, using Russell's no-class theory, introduces the notion of quasi-objects to speak of

\footnotetext{
${ }^{10}$ Frege "Comments on Sense and Denotation", [Frege PW] page 118.

11 Ibidem page 122 .

12 From Meaning and Necessity [Carnap MN] onward Carnap explained the relationships between intensions and extensions as for exemple in section 4 :

4.14 The extension of a predicator is the corresponding class

4.15 The intension of a predicator is the corresponding property

4. 13 Two predicators have the same intension iff they are logical equivalents
} 
classes. Classes are considered as the result of an iterative definition along the line of the theory of simple types and classes are quasi objets whose names have no independent meaning; they are just useful expedients to talk about the objects which satisfy a certain function. In this sense logical and mathematical objects (which are classes) serve just to fix conventions ${ }^{13}$.

A similar thesis is affirmed in 1934, but Carnap does not affirm anymore that classes are just useful expedients. Carnap defines in his book two languages, I and II. In both analyticity is defined through infinitary rules or through abstract concepts. In Language II, for example, Carnap defines the notion of valuation in order to define analyticity. The notion of valuation accomplishes the recursive attribution of values to the variables of the language. Such values are numerals, functions on numerals, classes of numerals. But in the case of predicates, the classes substituted in the valuation cannot be restricted to the classes definable in the language. Actually, according to the first incompleteness theorem, for every theorem of formal arithmetic there are properties which cannot be defined in the system, but for which we can exhibit a numeral possessing them. So the substitution accomplished through the valuation cannot properly be considered a substitution between a linguistic entity and a syntactical one. It is rather a substitution between a linguistic entity and an abstract one, taken from the abstract sets of all possible classes on numerals. The conventionalist turn of Logical syntax cannot be understood out of the defence that Carnap made of such a strategy. On $\S 34 \mathrm{~d}$ he stresses that the notion of "every property what-so-ever" (not restricted to the notion of "every property definable in $\mathrm{S}^{\prime}$ ) can be properly expressed in the logical syntactical language.

And this is all we need to use it. As Gödel noticed very clearly :

Carnap, to the objection that Platonism is implied by transfinite rules, replies that one may know how to handle the transfinite concepts without making any assumptions about the objective existence of the abstract entities concerned. This, of course, is true in the same sense as one also may know how to handle the concepts of physical objects without ascribing to these objects any existence in a metaphysical sense. ${ }^{14}$

This is the very conventionalist Carnap's rejection of Frege's thesis 3) : to admit abstract objects without any ontological engagement regarding them. It was on the consequences of such a conventionalist thesis that Gödel directed his criticism ${ }^{15}$.

\footnotetext{
13 See for ex. sections 33 and 107 of the Aufbau.

${ }^{14}$ [Gödel CWIII] page 33 .

15 Conventionnalism is indifferent to syntax or semantics. Even after the semantic turn Carnap reaffirms his rejection of any objective existence for mathematical objects and concepts. Classes are just mere "façon de parler", internal to a linguistic framework and deprived of any kind of non-linguistic existence. The fact that we use classes does not mean that we affirm their existence out of the linguistic frameworks of our theories.
} 
This conventionalist rejection of Frege's thesis 3) is strictly related to the rejection of any foundationalist perspective. We will come back to this point in section 2, but it has to be said here that the novelty of Carnap's solution can be appreciated only in relation to this rejection. Carnap does not consider the reduction of mathematics to language as a justification of mathematics. As Goldfarb clearly notices :

He could allow that, while mathematical truths come from syntactic or linguistic rules defining the framework, our recognition of particular truths or our trusting in them can require more mathematics or a different mathematics from that to which those rules yield ${ }^{16}$.

Carnap admits that the linguistic nature of mathematics (in the sense of it being just "framework truths") can be exhibited only from an infinite hierarchy of syntactic (or semantic) rules. Nevertheless this fact does not contradict the assertion of the linguistic nature of mathematics. An infinite hierarchy of syntactic or semantic systems is nothing but a hierarchical set of rules, and the use of these rules just presupposes the capability of manipulating symbols. That is just what is needed to explain the linguistic nature of mathematics. In finding such a solution Carnap saves the Fregean idea that the condition of possibility of mathematical knowledge is language. But he uses it in an empiricist direction: no structure of the knowing subject, no entity besides thos that language allows us to postulate, no objectivity but that reducible to the intersubjectivity of language, no necessity besides the necessity that language allows us to fix through the rules of language.

The price to pay for such a innovative solution is evident. Such an analysis cannot guarantee for mathematical and logical truths their necessity in the Fregean sense, even if it guarantees their a priori nature. Logical and mathematical truths are contingent in the sense that they can be revised if factors of efficiency, fruitfulness and simplicity ask for a revision. Carnap coherently accepts this consequence and explicitly affirms the possibility of revisig mathematical and logical truth, at least 15 years before Quine and his web metaphor ${ }^{17}$.

Besides these divergences, much of the Fregean analysis survives in Carnap's program. For Carnap as for Frege logic is a sort of metaphilosophy which provides a methodology for finding and organising, with due reflection, our considered judgements, at all levels of generality. For Carnap as for Frege, mathematics is the science of sciences. It is not concerned with the empirical world but with the concepts which are necessary to take account of the empirical world. Mathematics is a part of

\footnotetext{
16 [Gödel CWIII], page 329.

17 See [Carnap LSL] $\$ 82$.
} 
logic, the unifying language for all our knowledge. For Carnap as for Frege, language is the universal medium between us and the world. The universalism of the second is not betrayed by Carnap even by his Principle of Tolerance ${ }^{18}$. Universalism and apriority of logic and mathematics are saved, by conventionalism. This latter seems nothing but an extreme development of the conception of existence as conformity to laws, taking laws as hypothetical, conjectural language dependent, rejectible propositions.

\subsection{Logic and mathematics : Gödel}

To Carnap's ambitious project Gödel opposes a more traditional one, "rightward", as he calls it in his paper of 1961 . The central aims of this project are clearly to safeguard for mathematics the certainty, uniqueness and necessity of its knowledge, as well as restoring for reason of its power: for clear questions posed by reason, reason also finds clear answers ${ }^{19}$. These are Fregean desiderata but Frege's solution was no longer available for Gödel to sustain such a program. There are two reasons for that.

The first reason are the paradoxes. Frege isolated concepts and objects from propositions, through logical analysis. Such a strategy presupposes abstract entities as denotations for propositions: the True (for Frege from Sense and Denotation onward), or facts (for Russell). Such extravagant ontological presuppositions ${ }^{20}$ would be admissible if language, through propositions, allowed us to apprehend concepts and objects in a univoqual and clear way. But this is exactly what paradoxes show as impossible:: language allows us to speak of predicates which are not concepts and of concepts which are not classes, without helping to distinguish between paradoxical and unparadoxical predicates, concepts or classes. Language alone is not sufficient. Axiomatic theories are needed, that is, sets of propositions able to describe the way we can use such primitives. Axioms of a theory are not absolute unconditional laws, but conditional, conjectural hypotheses whose coherence has to be granted. Contrary to Frege and Carnap, Gödel is not a universalist. He never gave to language a central role in our knowledge -at least he never thought that language is the condition of possibilty of our knowledge.

\footnotetext{
18 Anytwo langauages can always be analysed and comparared in a common metalanguage, whose existence seems for Carnap an undoubtfull matter of fact, as he affirms for exemple in his answer to Beth in [Schilpp] page 929 : "Since the metalanguage ML serves as a means of communication between author and reader or among participants in a discussion, I always presupposed, both in syntax and in semantics, that a fixed interpretation of ML, which is shared by all participants, is given. This interpretation is usually not formulated explicitly; but since ML uses English words, it is assumed that these words are understood in their ordinary senses. The necessity of a this presupposition of a common interpreted language metalanguage seems to me obvious."

19 [Gödel CWIII] page 381.

${ }^{20}$ Gödel gave an explicit apreciations of such solution in the beginning of the paper on Russell.
} 
But then if the apprehension of primitives cannot be accomplished through language, how can we have experience of them? Intuition is invoked as the solution. Much has been said on the pre-Kantian, "rightward" nature of such a solution. It has less often been stressed that Gödel's intuition has very little to do with traditional notions of intuition. Gödel's intuition does not give, as classical rational intuition does, a direct knowledge of the primitives; it is neither intuitionistic intuition (Brouwer's primordial intution of time and of two-oneness, or Poincare's pure intuition of number) which determined uniquely the mathematical objects it allows us to construct. Gödel's intuition of the abstract primitives is not immediate, complete or deterministic. Gödel says that explicitly:

It should be noted that mathematical intuition need not be conceived of as a faculty giving an immediate knowledge of the objects concerned. Rather it seems that, as in the case of physical experience, we form our ideas also of those objects on the basis of something else which is immediately given. Only this something else is not, or not primarly, the sensations. That something besides the sensations actually is immediate given follows (independently of mathematics) from the fact that even our ideas referring to physical objects contain constituents qualitatively different from sensations or mere combinations of sensations, e.g., the idea of object itself, whereas, on the other hand, by our thinking we cannot create any qualitatively new elements, but only reproduce and combine those that are given. Evidently the "given" underlying mathematics is closely related to the abstract elements contained in our empirical ideas ${ }^{21}$.

There is no possibility to reduce the knowledge of these objects, empirical or abstract, to the intuition that we have of them. In this sense Gödel is exactly on the opposite of a reductionist positivist conception of science. Gödel's intuition gives only a guarantee of the existence of the logical and mathematical entities, a shadowy, imperfect, faillible guarantee of them, but nevertheless a guarantee. It is exactly such a guarantee that we need for the assumption of those entities, an assumption that is necessary for science, as he says in a very often quoted passage :

It seems to me that the assumption of such objects [mathematical objects] is quite as legitimate as the assumption of physical bodies and there is quite as much reason to believe in their existence. They are in the same sense necessary to obtain a satisfactory system of mathematics as physical bodies are necessary for a satisfactory theory of our sense perceptions and in both cases it is impossible to interpret the propositions one wants to assert about these entities as propositions about the "data", i.e., in the latter case the actually occurring sense perceptions. ${ }^{22}$

21 [Gödel CWII] page 268.

22 [Gödel CWII] Page 128. 
In the case of empirical objects Gödel's thesis is clear. We have sense data and the idea of object itself. This two elements give us the possibilty of the empirical objects whose knowledge can be attained only through the theory that we can form about them.

What does change for mathematics? Mathematical propositions do not express physical properties of the structures concerned in physics or in empirical sciences, but rather properties of the concepts with which we describe those structures. In the case of mathematics sense data are then the results of calculations. To the abstract idea of empirical object something corresponds: mathematical structures which are apprehended by a sort of sixth sense. According to Wang, Gödel affirmed that such a sixth sense must be closely related to the neural center of language, but notwithstanding independent of it :

7.3.5 I conjecture that some physical organ is necessary to make the handling of abstract impressions (as opposed to sense impressions) possible, because we have some weakness in the handling of abstract impressions which is remedied by viewing them in comparison with or on the occasion of sense impressions. Such a sensory organ must be closely related to the neural centre for language. But we simply do not know enough now, and the primitive theory on such questions at the present stage is likely to be comparable to the atomic theory as formulated by Democritus. ${ }^{23}$

This sixth sense frees objects and concepts from language. The question of their knowability (not of their beeing) is solved in a very Kantian mood. As Gödel says explicitly in the 1954 paper on the Continuum (footenote 40) :

Note that there is a close relationship between the concept of set explained in footnote 14 and the categories of pure understanding in Kant's sense. Namely, the function of both is "synthesis", i.e., the generating of unities out of manifolds (e.g., in Kant, of the idea of one object out of its various aspects). ${ }^{24}$

In Gödel view the "close relationship" is not to be taken as identity of function, as the unity of manifolds is not constructed by Gödel's intuition but only seen through the sixth sense.

The safeguard of the general characters of mathematics and of reason asks for such a solution. Reason can answer clear questions. The existence of objects whose knowledge is possible for us, and that can guarantee that facts are so and $\mathrm{so}^{25}$, has then been

\footnotetext{
23 [Wang]

24 [Gödel CWII ] page 268.

25 This seems to be the more convincing interpretation to such statements. Wang reports a very intresting quotation of Gödel in his [Wang]

7.3.1 If we begin with a vague intuitive concept, how can we find a sharp concept to correspond to it faithfully? The answer is that sharp concept is there all along, only we did not perceive it clearly.
} 
presupposed to ensure the possibility of such clear answers. Axioms are just conjectural hypotheses for the explication of phenomena. So the existence of the objects which these axioms presuppose and of the concepts which determines such objects can guarantee their coherence and their truth.

A natural question : can the facts concerning the result of such objects issued by this process (sense data+intuition) be considered as immediately given independently of any theoretical framework?

Concerning empirical facts, and in the context of his critics to Carnap, Gödel says explicitly that such empirical facts are empirical owing to the semantical rules of a language ${ }^{26}$.

Concerning abstract objects, the question is more complex, and we will not try to answer it here. Nevertheless it should be observed that, in the case of mathematics, the data (which are the bases on which intuition take the occasion to act) are calculations. Then it is clear that calculations are theoretical activities which have to be defined in theoretical frameworks.

The second reason why Frege's solution was not viable for Gödel is that Gödel is not an extensionalist. For him logic is the theory of concepts considered in intension and extension. Like Frege, Gödel respects the sharp distinction between the sense of a concept, the concept as denotation and the corresponding set or class. Against Frege he affirms that it is impossible to reduce concepts to their extensions. The theory of pure concepts is the central part of philosophy and it is impossible to reduce the theory of pure concepts to the theory of sets.

Paradoxes push him in such a direction. As we know, there are certain concepts whose ranges of applicability are not sets: for instance the concept of concept, the concepts of set of, the concept of being applicable to only one thing (or one object), the concept of being distinct from the set of all finite mathematical sets, the concept of being a concept of finite range, and so on. It is then erroneous to think that to each concept there corresponds a set. The range or the extensions of such concepts are mere classes, that is multitudes without any unity. Classes are only a derivative and hybrid convenience, introduced as a way of speaking about some aspects of concepts, and even if the

\footnotetext{
${ }^{26}$ We will come back later on this inequivocal statement on page 339 of [Gödel CWIII]. Anyway Gödel says about the so called factual sentences (and observe that he puts factual in very eloquent quotation marks) : "Moreover a rule about the truth of sentences can be called syntactical only if it is clear from its formulation, or if it somehow can be known beforehand, that it does not imply the truth or falsehood of any "factual" sentence (i.e., one whose truth, owing to the semantical rules of the language, depends on extralinguistic facts)." (the stress is our)
} 
principle of extensionality can be invoked for classes, it does not follow definitively for concepts.

8.6.9 Of course the axiom of extensionality holds for classes, because the façon de parler has been introduced for this purpose. In other words, two classes with the same members are identical. In contrast two concepts which apply to the same things are often different. Only concepts having the same meaning (intension) would be identical. ${ }^{27}$

This is the reason for invoking the meaning of concepts as the guarantee for the analyticity of the axioms of mathematics. Objects are not enough to cover mathematical concepts.

Summing up: Gödel refuses Fregean thesis 1) to each predicate corresponds a concept, to each concept corresponds a set which can be logically defined and 2) the extensionalist point of view on concepts. Gödel thinks that mathematics only has to do with concepts having sets as extensions. But logic is more than mathematics and a pure theory of concepts (intended in their intensional feature) must be possible even if in the actual state of knowledge we are very far from it.

Gödel totally accepts 3) not only for sets, but also for concepts. The logical and metaphysical existence of concepts and sets, independent from our capability to apprehend them, must be postulated if we want to restore those aspects of mathematical and logical truths such as necessity, uniqueness and certainty. Gödel affirms that our perception of concepts or of logical or mathematical objects can be refined exactly as our perception of physical objects. That means that even if our perception or intuition of such objects and concepts is fallible, as science is a fact, we can consider our scientific theory about them as approaching them more and more clearly. Truth for science is unique, eternal and not contextual. There is definitively for Gödel a fundamental agreement for describing by a set of axioms the concept of set. Actually, the present indetermination of the continuum problem shows that we do not yet have a complete axiomatisation. But the existence of such a coherent unique axiomatisation is garanteed by the existence of sets. Disjuncting sets from denotations of concepts, refusing to reduce intension to extension, rejecting the idea that language is the only condition of possibility of our knowledge and wanting to preserve in a strong sense the notion necessity for mathematics, Gödel is forced to adopt this odd idea of an internal sixth sense tied, as he says, to our capability of language but distinct from it.

\footnotetext{
27 [Wang] page 275.
} 


\section{Carnap, Gödel and Is mathematics Syntax of language?}

The main question we would like to answer in this second section is the following : is Gödel really blinded by his foundationalist point of view as, Goldfarb and Consuegra say? Is Gödel unable to appreciate the antifoundationalist turn that Carnap adopts from LSL onwards? It is possible to make this question more precise.

\subsection{Carnap and Gödel on foundationalism}

We will not deny here the evidence: Gödel and Carnap have antithetical conceptions of the task of logical analysis. The very content of Carnap's logical syntax has been precisely reconstructed in these last years of revival of studies on Carnap. After Gödel's incompleteness results Carnap had to redefine the notions of truth and consequence in order to

1) warrant truth by the virtue of language for logical and mathematical sentences.

2) abandon the program that Alberto Coffa calls "the Wittgenstenian program" for which the nature of mathematical truths implies their decidability, that is the possibility of knowing them through finitary linguistic procedures (by the way I will not engage myself with the real Wittgensteinien character of such theses. The finitistic thesis is just the interpretation that Carnap adopts of the Wittgensteinian analysis).

Carnap's technical solution relies on four methodological principles, three of which are made explicit in the discussion of the notion of explication contained in the first chapter of Logical Foundation of Probability. We can sum up these principles as follows :

A) the assertion of the linguistic nature of mathematical truths should not be intended as a foundation or a justification of these truths; on the contrary, such an assertion is the proposition of an explication of the importance and the use of mathematical concepts in science.

B) an explication proposes a transformation of such obscure notions as truth or consequence (the explicanda), which belong to a pre-formal step of science, in exact notions (the explicata) defined in a precise and rigorous language.

C) the explication is the proposition to replace, in scientific language, the vague and obscure explicanda with the precise and clear explicata. The explicata should be as similar as possible to the explicanda but this similarity does not imply the substitution in every context of the explicanda by the explicata. 
D) according to the Principle of Tolerance, we can use anything we want in the explication. In particular the language of the explicata can contain abstract concepts and infinitary rules; as we saw, it is actually possible to manipulate such abstract concepts and such infinitary rules without any hypostatisation and ontological engagement.

On the other hand Gödel :

a) thinks that the idea of an infinitary syntax is nonsensical. He believes that effectiveness, transparency and decidability are the fundamental features of Syntax.

b) is constant in his foundationalism. His conception of the task of logical analysis is not compatible with Carnap's idea of explication. Logical and philosophical analyses have to arrive at "fundamental conceptual advances" independently of the considerations of given languages or given linguistic fields.

Considering these fundamental divergences, the question is the following: does Gödel criticise Carnap's thesis from his own foundationalist point of view or does Gödel accept or assume in his argument Carnap's antifoundationalist thesis ?

\subsection{To replace/to reduce}

We will divide our analysis, in order to answer this question, into two parts. Firstly, we will consider the form of Gödel's argument, and then its content. The important differences between versions II and III of Is Mathematics Syntax of Language? on one side and versions IV and V on the other suggest that we look at it in this way. What is really different in these versions is Gödel's aim. It is very carefully specified in version II and III and less detailed in versions IV and V where Gödel seems much more concerned with specifying a positive theory of mathematical concepts then to present his pars destruens that he ever considered as definitive.

In version III (and II) Gödel considers three versions of the linguistic thesis, the thesis according to which mathematics is, or can be interpreted, as syntax (or semantics) of language.

First in section 1 he mentions the linguistic conception of Carnap, Schlick and Hahn around the thirthies at the Vienna Circle.

In section 4 he mentions Carnap's position of LSL .

In section 45, the linguistic thesis after Carnap's semantic turn is considered.

On the other hand versions IV and V treat all the versions of the linguistic thesis together.

Moreover in version III Gödel chooses his terms very carefully in describing in a precise way the three versions of the linguistic thesis. 
When he addresses himself to the Vienna Circle version of the thirties he says that according to it mathematics can be reduced to language. When he talks about Carnap's LSL Gödel says that according to it mathematical truths can be replaced by syntactic truths.

When he talks of the semantic version from 1939 onwards, he says that according to it, mathematical truths are just framework truths that is, they are true in virtue of the rules of the semantic metalanguage.

So the term 'to replace' is strictly reserved for the description of Carnap's $L S L$. This is very important if we consider that 'to replace', 'to take the place', 'to substitute' are the very terms used by Carnap's in Logical Foundation of Probability where the notion of explication is introduced.

\subsection{Explaining the nature of mathematics}

Now after these formal and terminological remarks, let's analyse the content of Gödel's argument. In version III Gödel specifies the way in which we have to understand the notions of Mathematics, Language, Syntax and Interpretation, the words used to express the linguistic thesis. He affirms here in particular about Syntax and Language the prerequisites of coherence and finitism.

Then he divides his argument into three parts to adapt his criticism first to the reductionist version of the thirties, second to the, let's say, explicationist version of LSL and finally to the last semantic version.

The six conditions which are listed and that could be subscribed to by a member of the Vienna Circle cannot be satisfied simultaneously and that suffices to refute linguistic reductionism because:

[...] only in that case [of the satisfaction of the six conditions] could a satisfactory foundation of mathematics be given independently of experience and without using mathematical intuition $[\ldots]^{28}$

Then at $\$ 25$ he considers the explicationist version and says :

[...] nor even the requirements 1-3, 5 (part 2), 6 [can be satisfied simultaneously] i.e. the axioms about the abstract transfinite concepts used in mathematics cannot be replaced by finitary considerations about combinations of symbols and their properties and relations, where "replacing" means that the same consequences as to ascertainable facts (to be more exact, the same universal propositions about them) can be derived in either case, and "ascertainable fact" here means any numerical equality for computable functions (such as $5+7=12$ ) or equivalent combinatorial relations ${ }^{29}$.

28 [Gödel] III, page 343. The stress is our.

${ }^{29}$ Ibidem, page 344 , The stress is our. 
That the program considered here is that of Carnap in $L S L$ can be proven (apart from the occurrences of the verb to replace) by the following consideration : the requisites 4 and 5 part 1 excluded by Gödel in this part of the argument correspond exactly to the conditions rejected by the liberalisation of the linguistic program.

In order to understand clearly the point we have to consider carefully what condition 4 and condition 5 part 1 , defined previously by Gödel, state;

4. Moreover a rule about the truth of sentences can be called syntactical only if it is clear from its formulation, or if it somehow can be known beforehand, that it does not imply the truth or falsehood of any "factual" sentence (i.e., one whose truth, owing to the semantical rules of the language, depends on extralinguistic facts).This requirement not only follows from the concept of a convention about the use of symbols, but also from the fact that it is the lack of content of mathematics upon which its a priori admissibility in spite of strict empiricism is to be based. The requirement under discussion implies that the rules of syntax must be demonstrably consistent, since from an inconsistency every proposition follows, all factual propositions included.

Condition 4 states a prerequisite that the systems of LSL do not satisfy. Language I and Language 2 of LSL are not demonstrably coherent. Carnap explicitly affirms that we can employ a system of syntactic rules even if we cannot prove their consistency within the system. Concerning his theorem 34.i24 which states that Language II is not contradictory, Carnap remarks that we should not overestimate the importance of the theorem. Theorem 34.i24 does not give us any assurance that Language II is really free from contradiction; actually to prove this theorem we need a language richer than that of language II, and the consistency proof of this metalanguage requires in turn an even richer language. The consistency of this latter is at least as doubtful as that of language II itself. This fits exactly with what we saw before; consistency can be afforded only by an infinite hierarchy of languages that become stronger and stronger.

So, when Gödel considers in section 25 the explicationist version of the linguistic thesis, he shows to have understood perfectly Carnap's position and to have taken it into account. He eliminates condition 4 from his pre-requisites because he admits that the syntactic rules chosen to explain the linguistic nature of mathematics cannot be demonstrably consistent, exactly as Carnap requires.

5.[... ] the phrase "Mathematics can be interpreted to be the syntax of language" will have to mean (1) that the formal axioms and the procedures of proof of mathematics can be deduced from suitably chosen rules of syntax, $[\ldots]^{30}$

\footnotetext{
${ }^{30}$ Ibidem page 339.
} 
Requisite 5 part 1 also states a condition that LSL does not satisfy. On one hand the proof of the analyticity for the axiom of choice and the axiom of induction, belonging to language II, need the use of these same axioms in the metalanguage. Carnap discusses the point at section 124 of LSL. On the other hand the proof procedures used for proving the analyticity of mathematics in language II are based on the notion of valuation which is not expressible in language II.

Once again, by eliminating this condition from the pre-requisites of the analysis of the explicationist point of view Gödel shows to have perfectly understood Carnap's idea. Consequently he admits that the syntactical systems which should show the linguistic nature of mathematics can be constructed with axioms and proof procedures which are not syntactical in the strict sense that Gödel gives to this notion.

\subsection{Summing up Gödel's argument}

We can now correctly interpret Gödel's argument in section 25. Let's suppose, Gödel says, we choose a system of rules to replace the vague notions of truth and of consequence in mathematics which is not syntactical in the strictest sense of this word; i.e. the chosen system of rules is not demonstrably consistent and uses infinitary rules, and axioms which are not syntactical or cannot be deduced by syntactical means. Could we then affirm that the linguistic program in the explicationistic version has succeeded ? Could we affirm that we have given a linguistic explanation of mathematical truths ?

Without any doubt we have obtained by this replacement explicit rules of use, such that, even if they cannot give a foundation of mathematics, allow us to describe the linguistic nature of mathematics in an appropriate and clear language.

Nevertheless, if mathematical activities have to be explained through the notion of convention, then if mathematical intuition has to be really replaced by the simple inferential activity from a conventional set of conventional rules, this explication and this replacement should lead to the same conclusions as to "ascertainable facts" which can be deduced using mathematics.

In other words the linguistic explication of mathematics should not destroy our trust in the predictive power of mathematics, predictive power both for pure mathematics and for applied mathematics. Using mathematical theorems we can, for example, predict certain properties of given numbers. Using the physical theory of elastic body, which can be formulated only using a certain portion of mathematics, we can predict whether a certain bridge, constructed according to these laws, falls down or not. Any trust in these 
predictions would be unjustified if the rules which allow us to formulate these predictions were simple conventions without content. Actually:

the scheme of the syntactical program to replace mathematical intuition by rules for the use of the symbols fails because this replacing destroys any reason for expecting consistency, which is vital for both pure and applied mathematics. ${ }^{31}$

The argument seems conclusive and not foundationalist. We have to justify the consistency of our rules just because we want to use them to make predictions. An explication which will not explain this trust will just be pragmatically inefficient because it would not explain the fruitfulness of our mathematics and its usefulness for us.

Why is this trust destroyed by the linguistic program ? What can explain our trust in the predictive power of mathematics?

i) empirical induction, based on the fact that no contradiction appeared until now. But then :

[...] it is true that, if consistency is interpreted to refer to the handling of physical symbols, it is empirically verifiable like a law of nature. However, if this empirical consistency is used, mathematical axioms and sentences completely lose their "conventional" character, their "voidness of content" and their "apriority" $[\ldots]^{32}$.

ii) mathematical intuition. This leads us to trust in the existence of mathematical concepts and objects. The trust in the existence of those entities, produces the trust in the properties of those entities (as no object could satisfy inconsistent properties). It is evident that this trust could be invalidated by the emergence of contradiction, because our intuition and our perception of mathematical objects and concepts are fallible. Notwithstanding, this trust in existence is a sufficient condition to explain our trust in the predictions which can be made using mathematics;

iii) a mathematical proof of consistency. But every mathematical proof of consistency will need transfinite or abstract concepts; if it is possible to prove the consistency of a system of rules in such a syntax, this proof, as Carnap admits, cannot warrant the consistency unless we admit the mathematical intuition of the abstract concepts and of the infinitary rules needed to produce the proof.

\footnotetext{
${ }^{31}$ Ibidem, $\S 29$ page 346 . The stress is our.

32 [Gödel CWIII] §20, page 342.
} 
Following the linguistic program, it is impossible to explain mathematical applicability.

We can certainly wonder if an explication or a description of the nature of mathematics should really explain or describe its applicability too. The answer seems to be negative. Nevertheless, such an explanation or a description of mathematics (without the explanation of mathematical applicability) will be at least as odd as an explanation or a description of soccer rules which would omit the fact that the players play to win and to put the ball inside the net.

What about the linguistic thesis after the semantic turn? The argument is essentially the same as for the syntactical version. It can be added that Gödel uses the parallel between physics and mathematics to show that there is no reason to answer differently way in the two areas. In $\$ 45$ of version V Gödel observes:

In general, if by using concepts and their relations among each other and with the sensations, one arrives at verifiable consequences, it is exactly from the existence of objects having these relations that the verifiable consequences follow. [...] That the existential assertions, also in mathematics are not mere "façon de parler" follows from the fact that they can be disproved (by inconsistencies derived from them) and that they have consequences as to ascertainable facts. [...] there is no reason to answer the question of the objective existence of mathematical and space-time objects differently.

\section{Moreover :}

If an inconsistency is not recognised as a disproval, but only as a proof for the "inexpediency" of the "convention" in question, the same can be done for laws of nature, which also can be interpreted to be conventions which become "inexpedient" in case a counterexample is met with. (version IV page 361)"

\section{Conclusion}

Besides the strength of Gödel's criticism, we can't accuse him of having overlooked the point. From the analysis of text III it seems clear that he does not take a foundationalist attitude to criticise a non foundationalist one. It seems also that Gödel is right in saying that from the linguistic point of view any explanation and description of mathematics won't be able to give an account of its applicability. Nevertheless it is coherent, even if odd, to deny that an explanation of the status of mathematics has to explain its applicability.

If Gödel's analysis is correct, if anyone thinks that such an explanation of the applicability is needed, he has only three solutions, if he wants to grant the differences between laws and norms : 
1) to admit the objective existence, independent of our constructions of mathematical concepts and objects. And to admit intuition and/or perception to grasp them.

2) to admit that mathematical concepts and objects are our constructions, primitive and irreducible to language (intuitionism). Then objectivity reduces to intersubjectivity.

3 ) to deny the objective (non-theoretical) existence of any object what-so-ever (empirical, physical or abstract) explaining the content of all our knowledge as a purely empirical content, and explaining our theory as the sophisticated product of our activity of reification. Mathematics needs sets. Sets have then an objective theoretical existence, but that existence has nothing to do with the truth of mathematics. Scientific theories are then highly conventional, not in Carnap's sense but in Poincaré's sense; that is: they are underdetermined in the face of experience.

The first solution is that of Gödel. The third can be represented by Quine. True sentences, observational and theoretical, are just the alpha and the omega of the scientific enterprise. They are related by structures, and objects figure as mere nodes of the structure. What particular objects there may be, for these theories, is indifferent to the truth of observation sentences, indifferent to the support of the theory in its predictions.

Quine's solution, nevertheless, has at least two main problems.

a) It assigned a content to mathematics insofar it is applied. The big range of modern mathematics which is not applied is there just there "to round the theory".

b) If the only content of mathematics is an empirical one, two theories can fit with the same empirical evidence, but they can have different ontologies. Should we use classical or intuitionistic mathematics, classical or intuitionistic logic ? Quine cannot avoid giving the disappointing answer that we have to use classical logic and classical mathematics just because in fact we use them.

Gödel's solution has evidently at least two problems.

a) The continuum problem. If we have reasonably tried all the possibility to find the extra axioms necessary to determine the answer to the questuion of the cardinality of the continuum, can we reasonably ask for objects seen by reason just to ensure us that such an axiom exists?

b) The question of analyticity.

As we saw intuition grants not only the coherence of axioms but also their analyticity and: 
"A proposition is called analytic if it holds 'owing to the meaning the concepts occurring in it', where this meaning may perhaps be undefinable, (i.e. irreductible to anything more fundamental)" CW II, page 139

The denotations, the concepts, cannot be given by a linguistical description, nor by simple intuition. The interesting paper of 61 in the third volume of the Collected Works, shows how Gödel considered phenomenology a promising method of clarification of this notion of meaning. He describe in 61 Husserl's method as

focusing more sharply on the concept concerned by directing our attention onto our own acts in the use of these concepts, onto our powers in carrying out our acts, etc. But one must keep clearly keep in mind that this pheomenology is not a science in the same sense as the other sciences. Rather it is [or in any cases should be ] a procedure technique that should produce in us a new state of conscoiusness in which we describe in detail the basic concepts we use in our tought, or grasp other basic concepts hitherto unknown to us. I believe there is no reason at all to reject such a procedure at the outset as hopeless.

For the moment nothing more can be said about these concepts than that there is in fact no reason to reject such a procedure.

[Carnap Au]

[Carnap LFP]

[Carnap LSL

[Carnap MN]

[Coffa]

[Consuegra]
R. Carnap, Der Logische Aufbau der Welt. Berlin-Schlachtensee : Weltkreis-Verlag, 1928.

R. Carnap, Logical Foundation of Probability. Routledge\& Kegan Paul LDT, 1951.

R. Carnap, The Logical Syntax of Language. Routledge\& Kegan Paul LDT, 1937. First edition Der logische Syntax der Sprache. Springer. 1934.

R.Carnap, Meaning and Necessity. The University of Chicago Press, 1956. Firt edition 1947.

A. Coffa, "Carnap, Tarski and the search of truth", Nô̂s 21, pages 547-572. 1987.

F. R. Consuegra, Kurt Gödel, Unpublished Philosophical Essays. Birhäusen Verlag. 1995. 
[Frege GG]

[Frege GGa]

[Frege GL]

[Frege KS]

[Frege PW]

[Gödel CW II]

[Gödel CW III]

[Heij]

[Schilpp]

[Wang]
G. Frege, Grundgesetze der Arithmetik, Band 1 and 2. Wissenschaftliche Buchgeselschaft Darmstadt, 1962. Firt edition Jena vol.1 1893. vol. 21903.

G. Frege, The basic Laws of Arithmetic (Translation and introduction by M. Furth) University of California Press, 1964.

G. Frege, Die Grundlagen der Arithmetik, eine logische matematische Untersuchung über den Begriff der Zahl (introduction by C. Thiel). Felix Meiner, 1986. First edition Breslau 1884.

G. Frege, Kleine Schriften (Edited by I. Angellelli). Georg Olms, 1990.

G. Frege, Posthumous Writings (Translated by P. Long, R. White, R. Hargreaves, Basil Blackwell, 1979.

K. Gödel, Collected Works, Volume 2, Publication s 1938-1974 (Edited by S.Feferman et alii)). Oxford University Press, 1990.

K. Gödel, Collected Works, Volume 3, Unpublished essays and lectures (Edited by S.Fefermanet alii)). Oxford University Press, 1995.

J. Van Heijenoort, Selected Essays. Bibliopolis. 1975.

The Philosophy of Rudof Carnap (Edited by A. Schilpp). The Library of Living Philosophers. La salle, 1963.

H. Wang, A Logical Journey, From Gödel to Philosophy. MIT Press, 1996 